\title{
Tailoring the gelatin/chitosan electrospun scaffold for application in skin tissue engineering: an in vitro study
}

\author{
Mohamad Pezeshki-Modaress ${ }^{1} \cdot$ Mojgan Zandi $^{2} \cdot$ Sarah Rajabi $^{3}$
}

Received: 20 June 2018 / Accepted: 12 August 2018 / Published online: 23 August 2018

(c) The Author(s) 2018

\begin{abstract}
The nanofibrous structure containing protein and polysaccharide has good potential in tissue engineering. The present work aims to study the role of chitosan in gelatin/chitosan nanofibrous scaffolds fabricated through electrospinning process under optimized condition. The performance of chitosan in gelatin/chitosan nanofibrous scaffolds was evaluated by mechanical tests, scanning electron microscopy (SEM), Fourier transform infrared (FTIR) and in vitro cell culture on scaffolds with different gelatin/chitosan blend ratios. To assay the influence of chitosan ratio on biocompatibility of the electrospun gelatin/chitosan scaffolds for skin tissue engineering, the culturing of the human dermal fibroblast cells (HDF) on nanofibers in terms of attachment, morphology and proliferation was evaluated. Morphological observation showed that HDF cells were attached and spread well on highly porous gelatin/chitosan nanofibrous scaffolds displaying spindle-like shapes and stretching. The fibrous morphologies of electrospun gelatin/chitosan scaffolds in culture medium were maintained during 7 days. Cell proliferation on electrospun gelatin/chitosan scaffolds was quantified by MTS assay, which revealed the positive effect of chitosan content (around 30\%) as well as the nanofibrous structure on the biocompatibility (cell proliferation and attachment) of substrates.
\end{abstract}

\section{Graphical abstract}
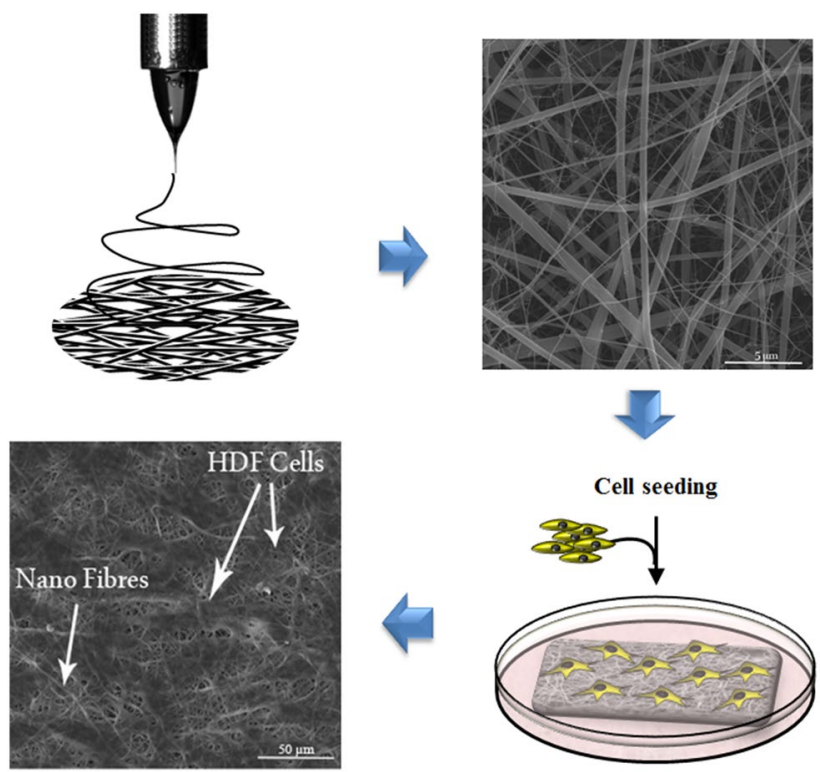

Keywords Gelatin/chitosan $\cdot$ Blend ratio $\cdot$ Nanofibers $\cdot$ Skin $\cdot$ HDF cells $\cdot$ In vitro

Extended author information available on the last page of the article 


\section{Introduction}

In recent years, electrospinning as a reliable technique for production of biomimetic scaffolds containing large network of interconnected pores has gained great attention in the literature (Bhardwaj and Kundu 2010; Dabouian et al. 2018; Pezeshki-Modaress et al. 2014; Saeed et al. 2017).

The human body tissue is composed of cells and extracellular matrix (ECM) which provide proper structural components as well as controlling the body processes, performances and wound healings (Sell et al. 2010). The ECM contains highly hydrated macromolecular networks such as collagen and glycosaminoglycans (Wang et al. 2007). Tissue engineering provides constructs appropriate for tissue substitution. A crucial factor in tissue engineering is to design and fabricate a biocompatible and biodegradable scaffold for culturing or hosting cells and transplanting into the body to regenerate the neo-organs (Pietrucha and Marzec 2005).

The cells have to interact with the scaffolds' structure in three dimensions. In natural ECM structure protein fibers' diameters are smaller than the cells and could provide a direct contact with the cells in three-dimensional orientations. In summary, the tissue-engineered scaffold should provide the opportunity for to exchange the signals between cells and the microenvironment and also between the cells in regeneration process (Barnes et al. 2007). Therefore, electrospunnanofibrous substrates are good candidates for using as tissue-engineered scaffolds with nanoscale structure (Heydarkhan-Hagvall et al. 2008). Many research works have focused on proteins as biopolymers for fabrication of nanofibrous scaffolds. The components of natural tissues, collagen and GAGs are widely used for scaffold fabrication which serves as efficient substitutes for native ECM (Mottaghitalab et al. 2015; Zhong et al. 2005). Gelatin is a natural biopolymer which is notably similar to collagen and still less susceptible to degradation during electrospinning process and enjoy a great potential to conduct the migration, adhesion, growth and organization of cells during regeneration process (Heydarkhan-Hagvall et al. 2008; Mahboudi et al. 2015; Pant and Kim 2013; Pezeshki-Modaress et al. 2013; Sadeghi et al. 2018; Zandi et al. 2007). Chitosan including glucosamine and $N$-acetylglucosamine is a biocompatible and biodegradable polymer and in vivo assays have proven that chitosan-based biomaterials show non-inflammatory reaction after injection, implantation and ingestion in the human body (Barikani et al. 2014; Baxter et al. 2013; Jayakumar et al. 2011; Mao et al. 2003a). Scaffolds containing chitosan also benefit other useful properties such as wound healing property (because of structural similarity to glycosaminoglycans), reducing scars, hemostasis, antifungal and bacteriostasis character, which make it applicable as a dermal scaffold. Therefore, using the blend based on gelatin and chitosan to improve their individual properties could be applicable as scaffolding materials in tissue regeneration (Esfandiarpour-Boroujeni et al. 2016; Martínez-Camacho et al. 2011; Modaress et al. 2012; Pezeshki-Modaress et al. 2013; Rahman et al. 2013). It has been reported that a higher ratio of gelatin $(>50 \% \mathrm{w} / \mathrm{w})$ in the gelatin/chitosan blended scaffolds resulted in better cell attachment and proliferation by considering the literature (Jafari et al. 2011; Modaress et al. 2012; Pezeshki-Modaress et al. 2013), but to the best of our knowledge there is no study on the influence of chitosan ratio on the nanofibrous scaffold properties in the literature. TFA/DCM (70/30) solvent system has been introduced as applicable solvent for electrospinning of gelatin/chitosan blends (Dhandayuthapani et al. 2010). Jafari et al. have fabricated gelatin/chitosan electrospun nanofibers using low molecular weight chitosan $\left(M_{\mathrm{w}} 1000 \mathrm{~g} \mathrm{~mol}^{-1}\right)$ and exhibited the potential of produced nanofibers for skin tissue engineering (Jafari et al. 2011). Pezeshki et al. introduced the optimized conditions for electrospinning process of gelatin/chitosan at TFA/DCM (70/30) solvent system using response surface methodology (Pezeshki-Modaress et al. 2014). In this work, nanofibrous structures of gelatin/chitosan blends were fabricated and the effects of chitosan ratio $(30,40,50 \mathrm{w} / \mathrm{w})$ on the chemical, physical and biological property of the obtained nanofibers were studied.

\section{Experimental}

\section{Materials}

Gelatin type B and chitosan of medium weight were purchased from Sigma-Aldrich (USA). $N$-(3-dimethylaminopropyl)- $N$ '-ethylcarbodiimide hydrochloride (EDC), trifluoroacetic acid (TFA), dichloromethane (DCM) and ethanol were purchased from Merck (Germany). DMEM/F12 medium, FBS, trypsin/EDTA, L-glutamine, and penicillin/streptomycin were purchased from Gibco, Canada.

\section{Preparation of polymer solution}

Chitosan solution of 5\% (w/v) and gelatin solution of $15 \%$ $(\mathrm{w} / \mathrm{v})$ were prepared by dissolving them in a co-solvent system of TFA and DCM (70:30) as previously reported (Pezeshki-Modaress et al. 2014). The two solutions were agitated overnight at room temperature to achieve homogeneous solutions with different ratios of 50/50,60/40 and $70 / 30$ (gelatin/chitosan). The solution was poured into a 5 $\mathrm{mL}$ syringe and was subjected to the electrospinning process using a horizontal system with a cylindrical collector 
covered by aluminum foil (Co881007 NYI, ANSTCO, Iran) at $30{ }^{\circ} \mathrm{C}$. Electrospinning was performed at $27 \mathrm{kV}$ applied voltage and $0.5 \mathrm{~mL} / \mathrm{h}$ flow rate. The distance between the needle tip and collector was $100 \mathrm{~mm}$. The electrospun nanofibers were kept at $4{ }^{\circ} \mathrm{C}$ and dry condition until further characterization.

\section{Morphologies of the fibers}

The morphology and diameters of electrospun nanofibers of gelatin/chitosan were investigated using scanning electron microscopy (SEM, VEGA, TESCAN, Czech) after gold sputter coating. The diameter of electrospun nanofibers was measured using image analysis software (Image J 1.42q, National Institute of Health, USA). At least 200 different fibers were used to determine the MFD and SDF.

\section{Measurement of porosity}

The average porosity of the electrospun gelatin/chitosan samples with different compositions was measured by liquid displacement technique (Jiankang et al. 2007; Modaress et al. 2012; Pezeshki-Modaress et al. 2017). Ethanol was selected as the displacement liquid since it permeates through the scaffolds without swelling or shrinking the matrix. The scaffolds (dry weight, $w_{\mathrm{d}}$ ) were immersed in ethanol for $30 \mathrm{~min}$ and the weights of the scaffolds in ethanol were recorded as $w_{1}$. The liquid on the surface of the scaffolds was removed by filter paper after taking out ethanol. The weight of the wet scaffolds was recorded as $w_{\mathrm{w}}$. The porosity of the gelatin/chitosan scaffolds was obtained by

Porosity $(\%)=\left(W_{\mathrm{w}}-W_{\mathrm{d}}\right) /\left(W_{\mathrm{w}}-W_{1}\right) \times 100$

The values are expressed as the means \pm standard error $(n=3)$.

\section{Mechanical properties}

The tensile mechanical properties were tested with a mechanical tester (SANTAM, STM-20, Iran). The samples were rectangular disks $\left(30 \times 10 \mathrm{~mm}^{2}\right)$ with a thickness of around $40 \mu \mathrm{m}$ tested at a constant tensile deformation rate of $5 \mathrm{~mm} / \mathrm{min}$ in the dry state at room temperature. The stress and elongation-at-break were determined. The values are expressed as the means \pm standard error $(n=3)$ (Mao et al. 2003b; Modaress et al. 2012).

\section{Fourier transform infrared (FTIR) analysis}

The chemical structure of the gelatin/chitosan nanofibers was analyzed by Fourier transform infrared spectroscopy (FTIR) using a BRUKER FTIR spectrophotometer
(EQUINOX 55, Germany). The infrared spectra of the samples were measured over a wavelength range of $4000-400 \mathrm{~cm}^{-1}$.

\section{Crosslinking and sterilization}

The nanofibrous scaffolds were chemically crosslinked using $0.02 \mathrm{~g} N$-(3-dimethylaminopropyl)- $N$ '-ethylcarbodiimide hydrochloride (EDC) (Merck, Germany) in $10 \mathrm{~mL}$ pure ethanol for $24 \mathrm{~h}$, and then sterilized with $70 \%$ ethanol for $4 \mathrm{~h}$. They were rinsed several times in phosphate buffer solution (PBS) to remove traces of ethanol. The cross-linked electrospun scaffolds were kept at $4{ }^{\circ} \mathrm{C}$ under dry condition for further assessment.

\section{In vitro human dermal fibroblast cell culture}

HDF cells were cultured in DMEM/F12 medium containing $10 \%$ fetal bovine serum (FBS), $1 \%$ penicillin/streptomycin and $1 \% \mathrm{~L}$-glutamine in T-75 flask tissue culture. Four and six passage cells were used in all the experiments. The cells were cultured at $37{ }^{\circ} \mathrm{C}$ and $5 \% \mathrm{CO}_{2}$. The culture medium was refreshed every $72 \mathrm{~h}$. At confluence, fibroblast cells were harvested and subcultivated in the same medium. The cells were separated with $0.05 \%$ trypsin/EDTA, centrifuged, and re-suspended in medium. The sterile nanofibrous scaffolds were incubated in culture medium overnight in order to check contamination, increase protein adsorption and cell attachment onto the nanofibers. The density of $1 \times 10^{4}$ cells/ $\mathrm{cm}^{2}$ in culture medium (DMEM/F12, Gibco, Canada), containing $10 \%$ fetal bovine serum, was seeded on the scaffold in a 24-well culture plates. The medium was replaced regularly every $48 \mathrm{~h}$; the culture process was carried out in an incubator at $37^{\circ} \mathrm{C}$ with $5 \% \mathrm{CO}_{2}$. All experiments were run in triplicate. Cell proliferation on film served as reference and control substrates. The samples were analyzed by DAPI staining, MTS and scanning electron microscopy (SEM).

\section{MTS Assay}

To evaluate the cell proliferation and metabolic activity on scaffolds, the MTS (Promega, G5421) assay was performed according to the manufacturer's instructions. Briefly, HDF cells were seeded at a density of $1 \times 10^{4}$ cells $/ \mathrm{cm}^{2}$ on scaffolds. The medium was changed every 2 days. At days 1 , 3, 7 and 14, the scaffolds were transferred into new wells and the MTS solution was added into each well, after which the plates were incubated in the dark at $37{ }^{\circ} \mathrm{C}$ for $3 \mathrm{~h}$. The absorbance of the solution was measured at $490 \mathrm{~nm}$. The experiments were run in triplicate. 
Fig. 1 SEM micrographs of nanofibrous scaffolds fabricated using $0.5 \mathrm{~mL} / \mathrm{hr}$ flow rate and $27 \mathrm{kV}$ applied voltage. a Chi 50, b Chi 40, c Chi 30
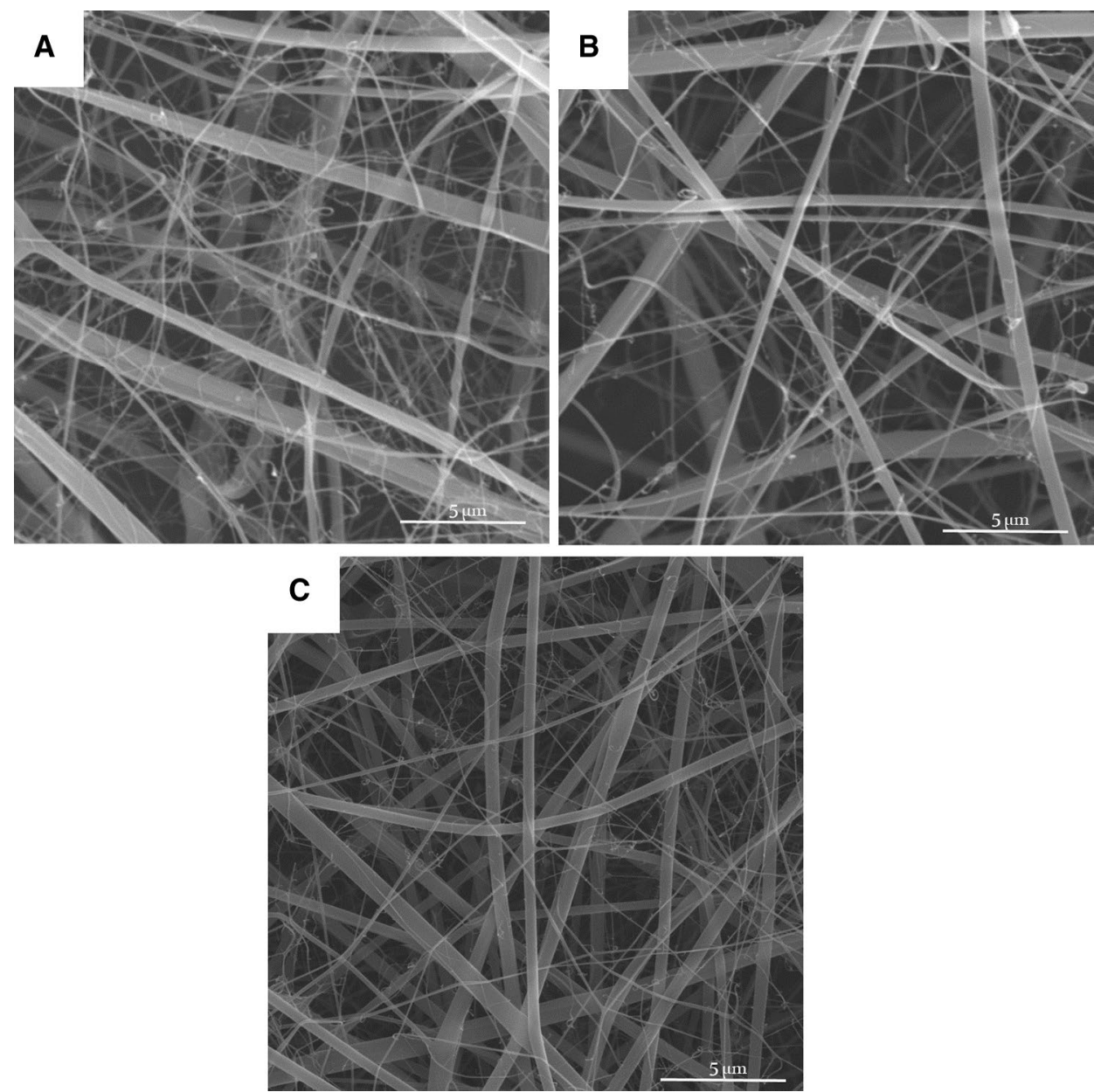

\section{Scanning electron microscopy and DAPI staining}

As far as the study of the morphological characteristics of cells cultured onto the nanofibrous matrices and also maintaining the fibrous structure of gelatin/chitosan substrate at cell culture medium were concerned, SEM observations were carried out. The morphological characteristics of the cells cultured onto the nanofibrous matrices were studied through scanning electron microscopy. After growing for 1 and 7 days, the cellular constructs of the HDF cells were harvested, washed with PBS to remove non-adherent cells and then fixed with $2.5 \%$ glutaraldehyde overnight at $4{ }^{\circ} \mathrm{C}$, dehydrated through a series of graded alcohol solutions (50, $70,80,90,95$ and $100 \%$ ) and then vacuum-dried overnight. Dry cellular constructs were sputter coated with gold and observed by SEM at an accelerating voltage of $15 \mathrm{kV}$. For DAPI staining process, samples were fixed for $2 \mathrm{~h}$ in a $10 \%$ PBS/neutral-buffered formalin solution ( $\mathrm{pH} \mathrm{7.4)}$ at $25^{\circ} \mathrm{C}$. Subsequently, samples were washed in d.d. $\mathrm{H}_{2} \mathrm{O}$ and dehydrated in a graded alcohol series. Then, the samples were stained with 4,6-diamidino-2-phenylindole (DAPI, SigmaAldrich, D8417), after which they were visualized utilizing
Table 1 The MFD and SDF of electrospun gelatin/chitosan scaffolds

\begin{tabular}{lll}
\hline Sample & MFD (nm) & SDF (nm) \\
\hline Chi 50 & 180 & 190 \\
Chi 40 & 196 & 185 \\
Chi 30 & 185 & 180 \\
\hline
\end{tabular}

an Olympus fluorescent microscope (BX51 with Olympus DP72 digital camera).

\section{Results and discussion}

\section{Morphology of the scaffolds}

The nanofibrous structure of gelatin/chitosan blends scaffolds with suitable properties was prepared using electrospinning technique. Scaffolds with four different gelatin/ chitosan blend ratios of 100/0, 70/30, 60/40 and 50/50 were fabricated and the influence of chitosan ratio on chemical, physical, mechanical and biological properties of the scaffolds was investigated. Figure 1 shows SEM micrographs of 
the electrospun gelatin/chitosan scaffolds containing different ratio of chitosan. All scaffolds have nanofibrous structure and no beads were observed. The mean fiber diameter and their standard deviation of fabricated scaffolds are listed in Table 1.

\section{Physical and mechanical properties of gelatin/ chitosan scaffolds}

The dermis regeneration process would be achieved when nutrients penetrate into the scaffold through interconnected pores and the exudation of the wound can be steadily absorbed by the scaffold. The structures with high porosity and pore interconnectivity not only improve mass transfer of oxygen and nutrients into the inner pores, but also efficiently remove metabolic products (Jiankang et al. 2007; Pezeshki-Modaress et al. 2013). The porosity of the electrospun gelatin/chitosan scaffolds containing different ratios of chitosan is illustrated in Fig. 2. The preferred porosity of scaffolds used for tissue engineering should generally be within the range of $60-90 \%$ (Chandrasekaran et al. 2011; Chong et al. 2007). The porosity of the electrospun gelatin/ chitosan scaffolds achieved in this study was around $90 \%$, comparable to the scaffold fabricated from conventional scaffolding techniques such as phase separation, salt leaching and fiber bonding and also at preferred porosity range of scaffolds used for tissue engineering (Chandrasekaran et al. 2011; Chong et al. 2007). One of the main advantages of the electrospun nanofibrous scaffolds is their highly interconnected pores which are created by nanofibers lying loosely upon each other. The nanofibrous network structure fabricated using electrospinning technique possess interconnected pores, which are created by nanofibers lying loosely upon each other, best mimics the natural ECM and improves its application as tissue engineering scaffolds. The obtained results reveal that all the prepared electrospun samples are highly porous and there is no significant difference between the electrospun samples.

The high degree of porosity and interconnected pore morphology (as illustrated in Figs. 1,2) of electrospun scaffolds fabricated in this study could provide good nutrient and oxygen transfer throughout the porous scaffolds, which results in better cell migration and regulating ECM formation. The mechanical performance of scaffolds in skin tissue engineering, subjected to tensile stresses and has high area/thickness ratio, is an important factor that can influence the clinical operation and wound healing. The mechanical property of nanofibrous scaffolds with different chitosan ratios is illustrated in Fig. 3. The results reveal that the tensile strength and elongation-at-break of all three nanofibrous scaffolds were more than $1 \mathrm{MPa}$ and $2.7 \%$, respectively. The same as porosity, the mechanical property of three samples was not statistically different.

\section{Chemical analysis}

The chemical structure of fabricated gelatin/chitosan nanofibers was investigated using FTIR spectroscopy analysis. Representative spectra for blended samples, as well as gelatin (Chi 0) and chitosan (Chi 100) electrospun nanofibers in the wave number range of $400-4000 \mathrm{~cm}^{-1}$, are shown in Fig. 4. All nanofibers' spectra exhibited peaks at $3330 \mathrm{~cm}^{-1}$ for $-\mathrm{NH}_{2}$ and $-\mathrm{OH}$ stretching vibration and $3100-2900 \mathrm{~cm}^{-1}$ for $\mathrm{C}-\mathrm{H}$ aliphatic group stretching vibration $\left(3100 \mathrm{~cm}^{-1}\right.$ for alkenyl $\mathrm{C}-\mathrm{H}$ stretch and $2970 \mathrm{~cm}^{-1}$ for $\mathrm{CH}_{2}$ asymmetrical stretching).

The electrospun gelatin (Chi 0) spectrum illustrated several characteristic absorption bands at $1670 \mathrm{~cm}^{-1}$ for amid $1(\mathrm{C}=\mathrm{O})$ stretching vibration, $1550 \mathrm{~cm}^{-1}$ for amid $2(\mathrm{~N}-\mathrm{H})$ bending vibration, $1465 \mathrm{~cm}^{-1}$ for $\mathrm{CH}_{2}$ bending, $1262 \mathrm{~cm}^{-1}$ for amid $3(\mathrm{C}-\mathrm{N})$ stretching vibration and $1160 \mathrm{~cm}^{-1}$ for $-\mathrm{C}-\mathrm{O}$ stretching (Al-Saidi et al. 2012; Bin Ahmad et al. 2011; Lai et al. 2012; Nguyen and Lee 2010). The
Fig. 2 The porosity of electrospun scaffolds containing different ratio of chitosan

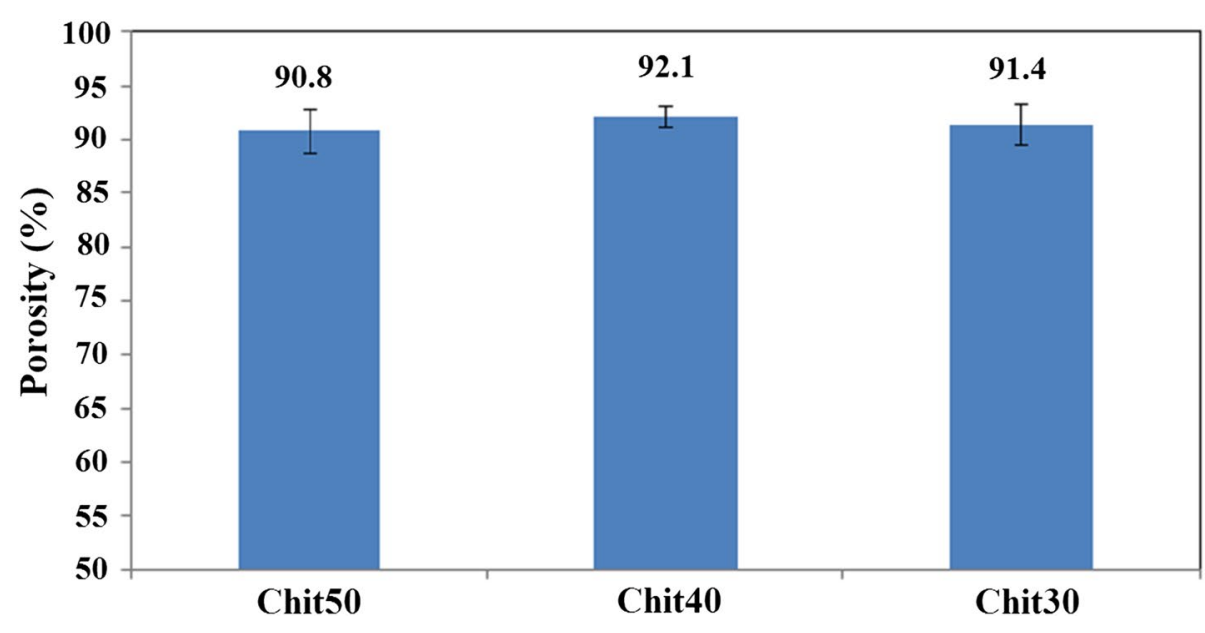

Springer 
Fig. 3 The mechanical property of gelatin/chitosan electrospun scaffolds
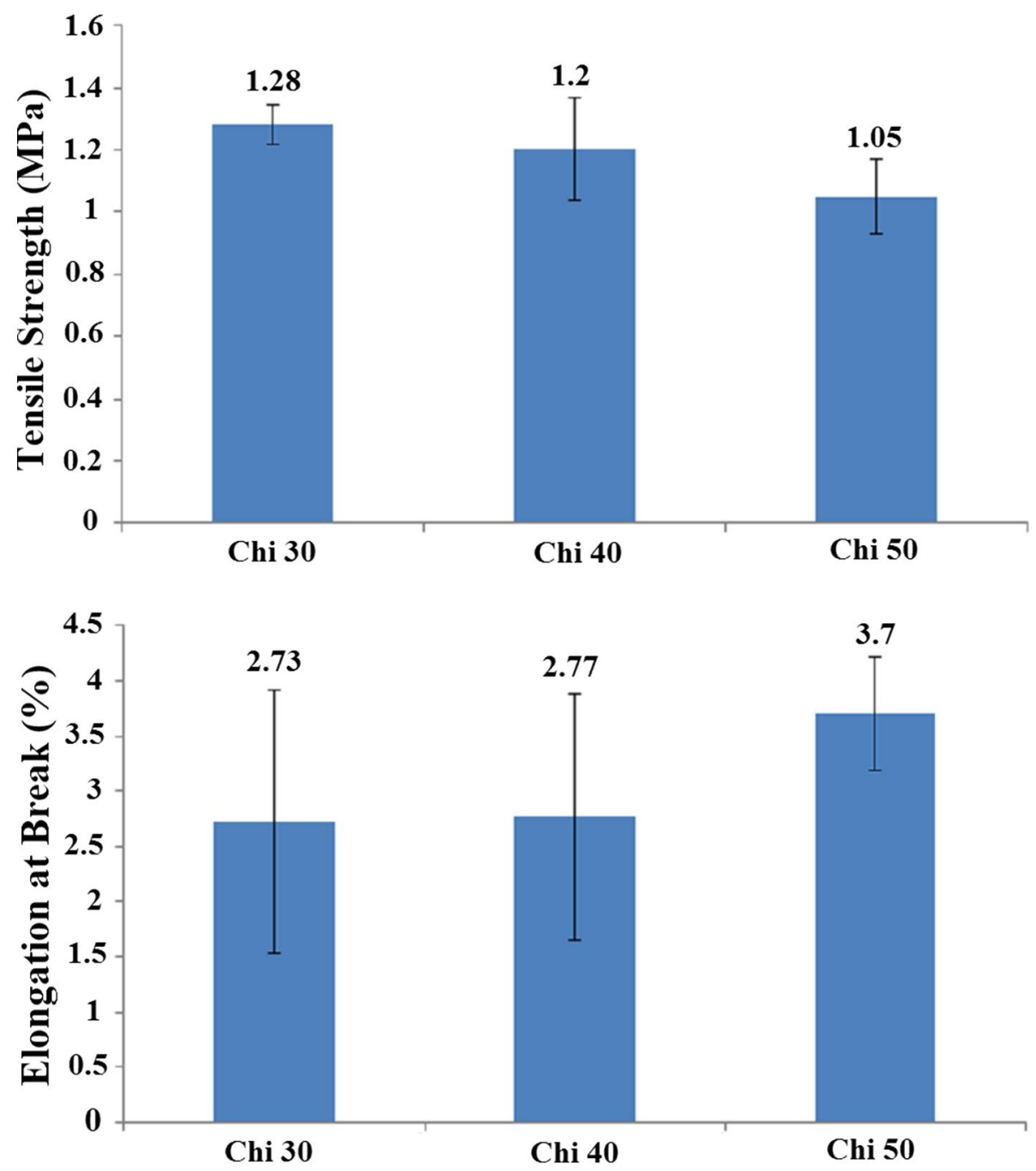

Fig. 4 The FTIR analysis of gelatin/chitosan electrospun scaffolds containing different ratio of chitosan: a) Chi $0 \mathrm{~b}$ ) Chi 30 c) Chi 40 d) Chi 50 e) Chi 100

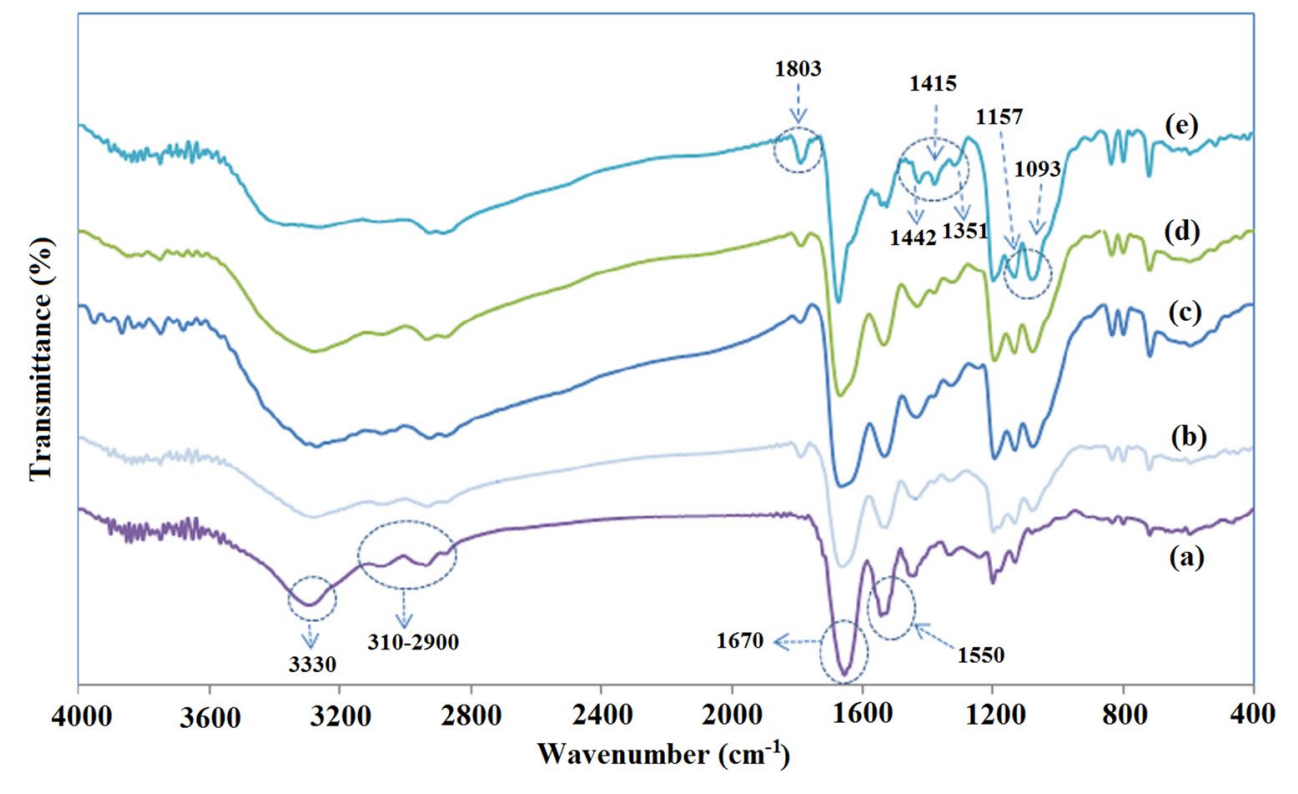


electrospun chitosan (Chi 100) spectrum exhibited strong peak at $1680 \mathrm{~cm}^{-1}$ with a shoulder around $1645 \mathrm{~cm}^{-1}$ and a peak at $1566 \mathrm{~cm}^{-1}$ for $\mathrm{C}=\mathrm{O}$ stretching vibration, vibration of amine group and ammonium ions, respectively (Haider et al. 2010; Qian et al. 2011). The peak at 1803 represents CO-F group. The Chi 100 spectrum displayed also some peaks at 1442,1415 and $1351 \mathrm{~cm}^{-1}$ for $\mathrm{C}-\mathrm{H}$ bending vibration, 1093 and $1157 \mathrm{~cm}^{-1}$ for $\mathrm{C}-\mathrm{O}$ stretching vibration (Bin Ahmad et al. 2011; Dhandayuthapani et al. 2010; Yin et al. 2003).

The FTIR spectra of the nanofibrous gelatin/chitosan blends exhibited characteristic absorption bands at 1680 and $1554 \mathrm{~cm}^{-1}$ representing the carboxyl and amine groups.

The shifting and broadening of both 1680 and $1554 \mathrm{~cm}^{-1}$ peaks for gelatin/chitosan blend spectrum in comparison of pure gelatin and chitosan spectrum revealed the formation of hydrogen bonding between chitosan and gelatin. The hydroxyl, carboxyl and amine groups of gelatin could form hydrogen bond with hydroxyl and amine groups of chitosan. These interactions could lead to polyanionic-polycationic complex (Bin Ahmad et al. 2011; Dhandayuthapani et al. 2010; Qian et al. 2011). The gelatin/chitosan blends spectrum show absorption bands at 1093 and $1415 \mathrm{~cm}^{-1}$ representing the $\mathrm{C}-\mathrm{O}$ stretching (represent the saccharide structure of chitosan) and $\mathrm{C}-\mathrm{H}$ bending vibration, respectively, which is absent in pure gelatin spectrum. There is another important peak at $1803 \mathrm{~cm}^{-1}$ in Chi 100 and gelatin/chitosan blends spectrum for $\mathrm{CO}-\mathrm{F}$ groups which is absent in pure gelatin spectrum.

\section{In vitro cell adhesion and proliferation}

The cell biocompatibility of the gelatin/chitosan nanofibrous scaffolds was investigated in vitro by inspecting the adhesion, spreading and proliferation of HDF cells onto electrospun scaffolds. HDF Cells bioactivity on nanofibers in terms of attachment and proliferation are fundamental to evaluate nanofibers capability as promising scaffolds which is achieved using DAPI staining, SEM observation and MTS
Fig. 5 DAPI staining of HDF cells on electrospun gelatin/chitosan scaffold after 1 and 7 days of culturing. a Chi 50 b Chi 40 c Chi 30
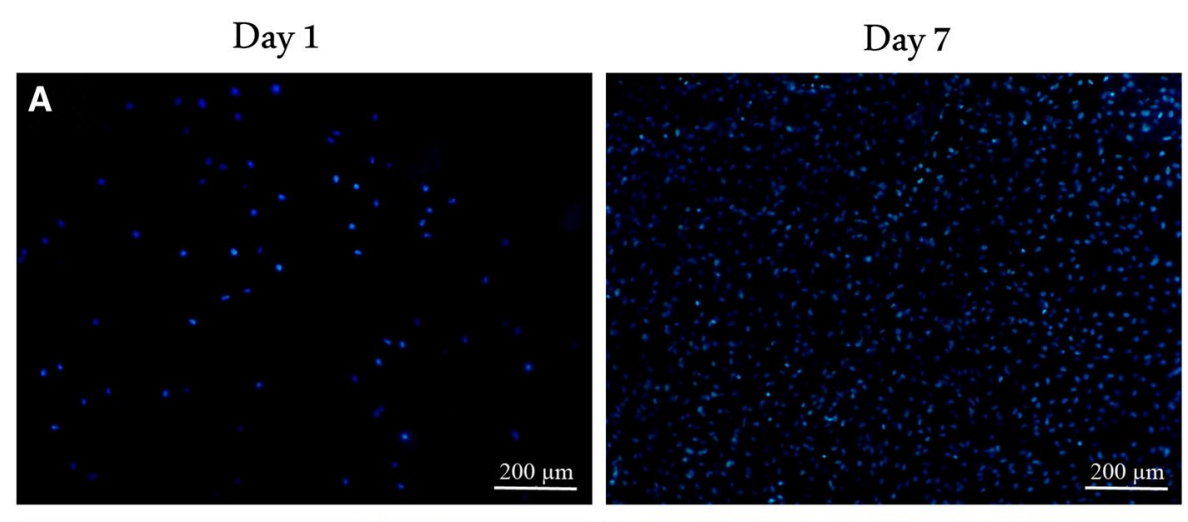

B
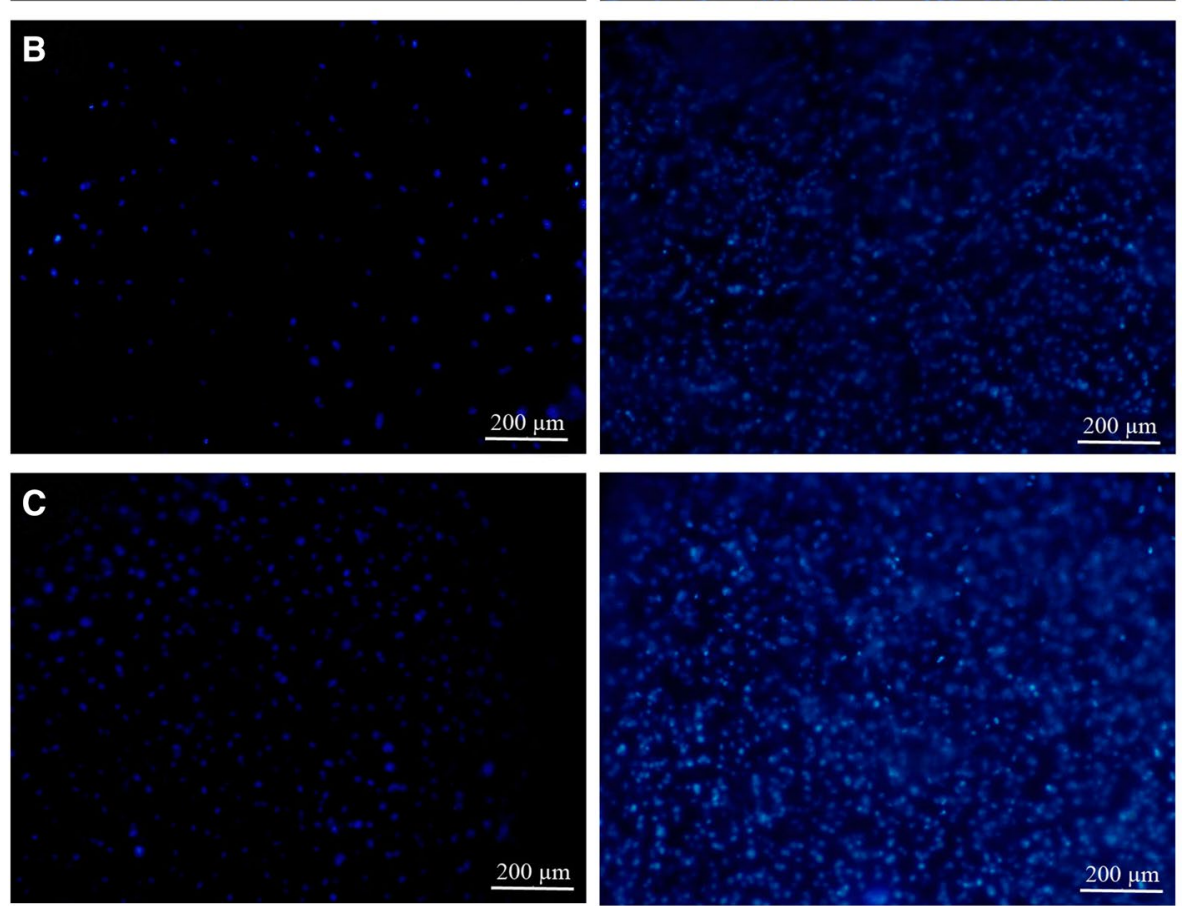
assay. The DAPI staining results are shown in Fig. 5 at days 1 and 7 , which reveal the good attachment and proliferative behavior of cells on all three nanofibrous scaffolds; however, the Chi 50 shows lower cell attachment in comparison with other samples. The SEM observation was used to investigate the cell interaction accurately with the nanofibrous substrates at day 1 and 7 to fulfill the DAPI staining results. Figures 6, 7 and 8 represent the micrographs of HDF cells cultured on electrospun scaffolds containing different ratios of chitosan which display the high HDF cells attachment and well spreading on all three nanofibrous scaffolds with spindle-like shape and complete stretching morphology. Comparison of micrographs at days 1 and 7 reveals the population of cells and shows consistency with DAPI staining results. The results of SEM observation at day 1 reveal that the HDF cells interacted well with the surrounding fibers and attached to the surfaces byfilopodia. The HDF cells adhered on gelatin/chitosan nanofibers and demonstrated the characteristic spindle-like shape of HDF cells, signifying that the gelatin/chitosan nanofibers maintained the phonotype of fibroblast cells. The neighbor cells were interconnected by excreted filopodia which could be observed in SEM micrographs. The observation at day 7 reveals the cell migration, proliferation and also the formation of a continuous monolayer covering the surface of nanofibers by interconnection of adjacent cells. Comparison of Fig. 9a, b clearly displays the population of cells during 7 days on Chi 30. These results also demonstrated that electrospun gelatin/chitosan scaffolds have maintained its nanofibrous morphology for 7 days at cell culture media employing suitable crosslinking conditions. Because of discrepancy in the hydrophilicity, chemical composition and charge of proteins such as gelatin and polysaccharides like chitosan (Chen et al. 2010; Pezeshki-Modaress et al. 2013; Zhong et al. 2005), electrospun gelatin/chitosan scaffolds containing different amount of chitosan (as a positively charged polysaccharide) were expected to reveal different cellular behavior. The HDF cell proliferation on electrospun gelatin/chitosan nanofibrous scaffolds was quantified by MTS assay for 14 days (1, 3, 7 and 14 days). The electrospun gelatin (Chi 0 ) was used as control for evaluating the role of chitosan in the HDF cellular activity. To investigate the influence of substrate nanotopography on cells population, we have used a gelatin/ chitosan cast film as second control which was fabricated from the same solution that was used for the preparation of Chi 40 sample.

As shown in Fig. 10, in 14 days of HDF cells culturing, the cell number increased with culture time on all substrates
Fig. 6 Morphology of HDF cells on electrospun Chi 50 scaffold after $1(\mathbf{a}, \mathbf{b})$ and 7 (c, d) days of culturing with different magnification
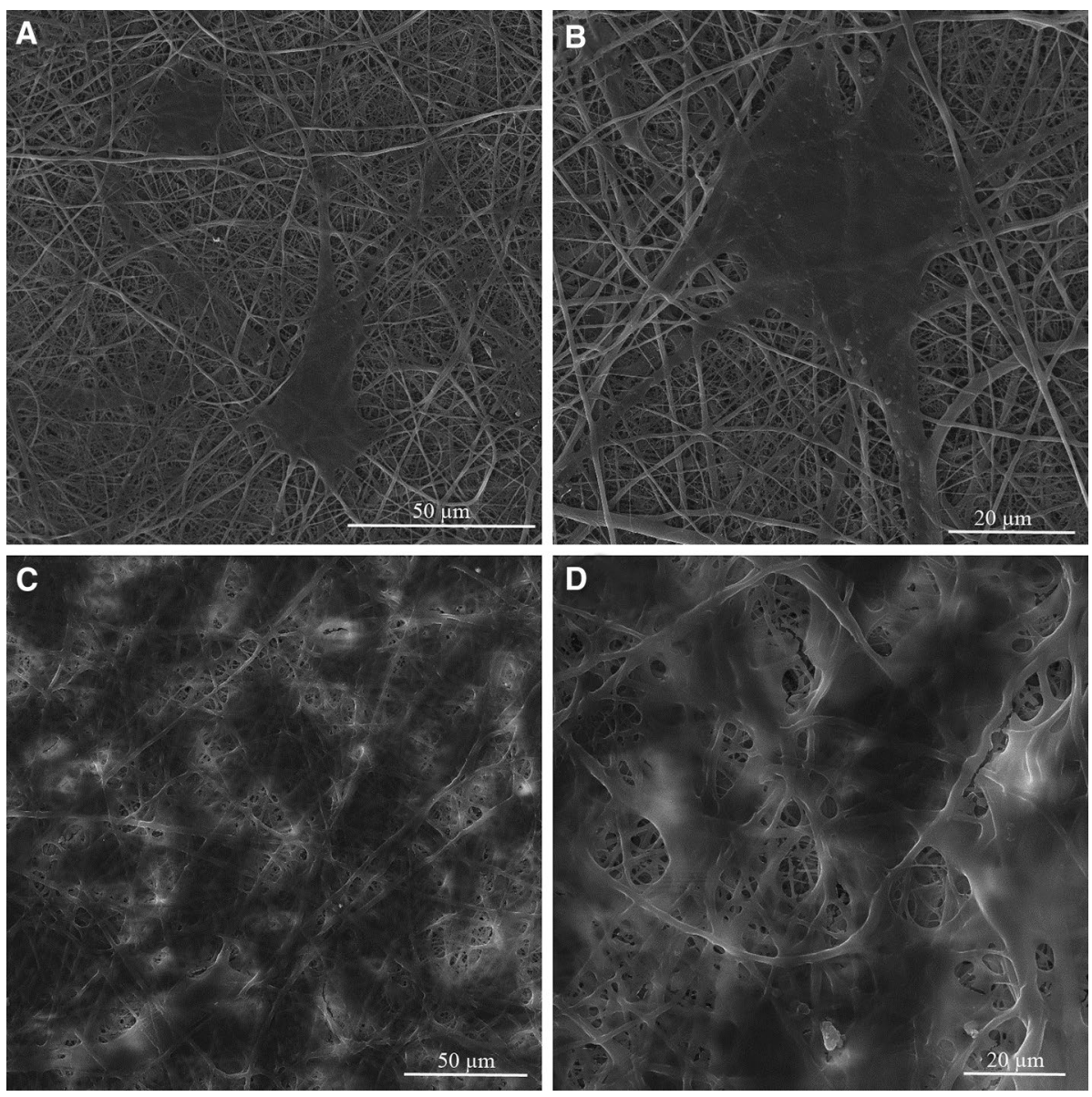
Fig. 7 Morphology of HDF cells on electrospun Chi 40 scaffold after $1(\mathbf{a}, \mathbf{b})$ and 7 (c, d) days of culturing with different magnification
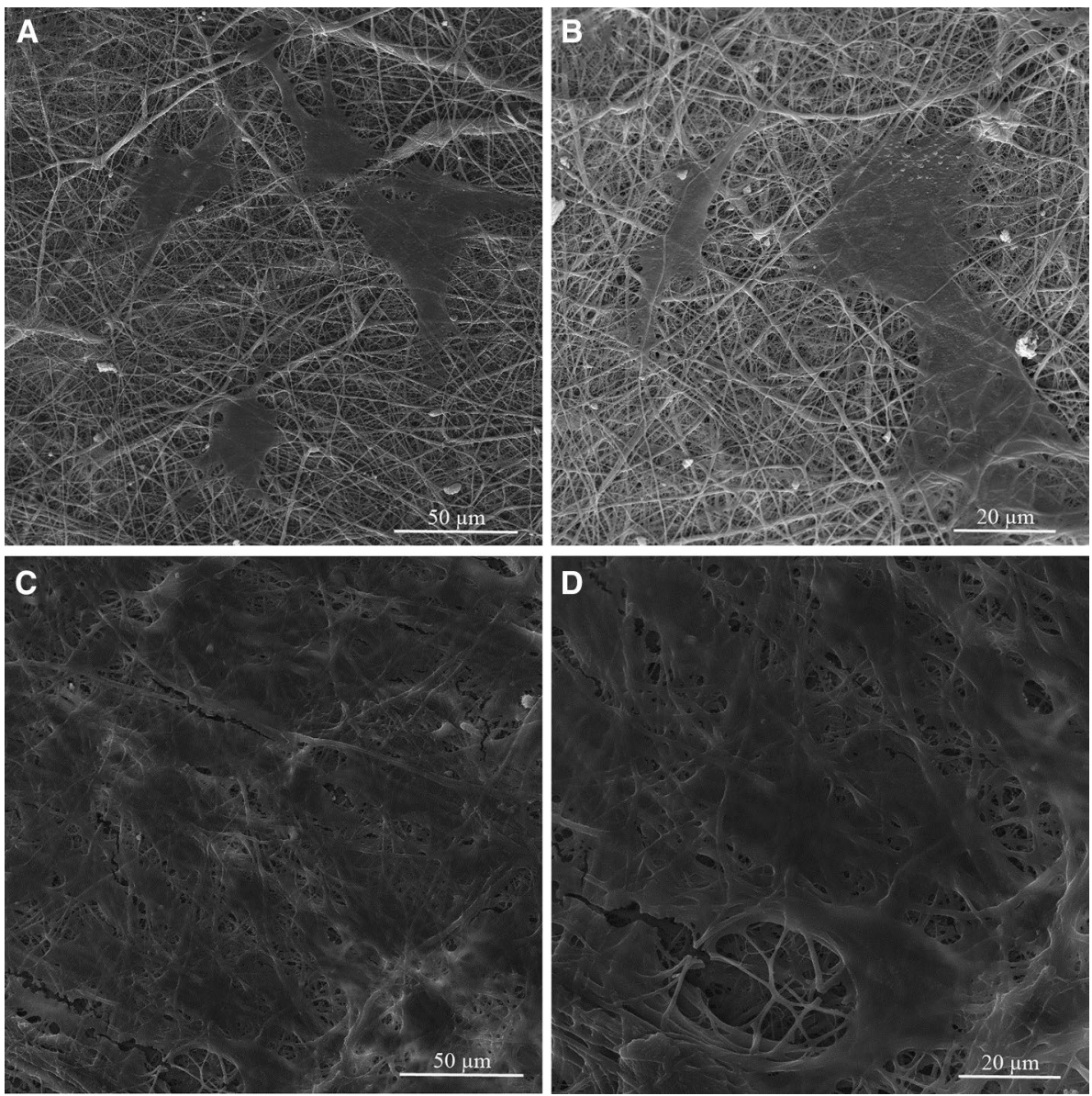

groups. On days 1 and 3, no statistically difference in proliferation of cells on the substrates could be observed except the Chi 0 which exhibited low cell proliferation. At 7 and 14 days, the substrates performance for proliferation of HDF cells could be observed more clearly. The nanofibrous scaffolds Chi 40 and Chi 30 show the best performance for HDF cell proliferation. When the cell proliferation between the Chi 40 electrospun scaffold and gelatin/chitosan (60/40)cast film was compared, the HDF cells response much better on the nanofibrous scaffold which reveal the potential of electrospinning method. When the cell proliferation of the Chi 0 nanofibrous scaffold is compared with Chi 30, 40 and 50 nanofibrous scaffolds, it is confirmed that the chitosan presence in the gelatin structure has improved the proliferation rate of HDF cells. Our results demonstrated that using nanofibrous structure and the presence of polysaccharide molecules (chitosan) in blend with gelatin would be useful for cellular behavior of substrates. Moreover, the presence of $40 \%$ polysaccharide in composition of blends shows higher influence than substrate morphology on the cells proliferation considering the lowest cells proliferation for Chi 0 . These results signify that there is a delicate relationship between chitosan and gelatin ratio for responding to the HDF cells. It seems that the low chitosan containing scaffolds (Chi 30) might have accelerated adhesion and proliferation of fibroblast cells $(p<0.05)$. It has been stated that incorporation of polysaccharides like chitosan or glycosaminoglycan into the proteins like collagen and gelatin not only increases their mechanical properties, but also improves their cellular activity (Chen et al. 2010; Mahboudi et al. 2015; Pezeshki-Modaress et al. 2013; Zhong et al. 2005). In this work from the in vitro study of HDF cells behavior on gelatin/chitosan nanofibers, it was established that the protein-polysaccharide blends and also nanofibrous morphology with high porosity favor cell attachment and proliferation by providing a three-dimensional extracellular environment. Advancement in fibroblast cells attachment to the fibers and also high proliferation rate on electrospun gelatin/chitosan scaffolds indicate high bioactive properties achieved with blending gelatin and chitosan in nanofibrous form and molecular interactions created between the gelatin, chitosan and HDF cell membrane.

\section{Conclusion}

Based on our results, gelatin/chitosan nanofibrous scaffolds were fabricated using electrospinning at optimized condition with average fiber diameter in the range 180-196 nm. The fibrous morphologies of electrospun gelatin/chitosan 
Fig. 8 Morphology of HDF cells on electrospun Chi 30 scaffold after $1(\mathbf{a}, \mathbf{b})$ and 7 (c, d) days of culturing with different magnification
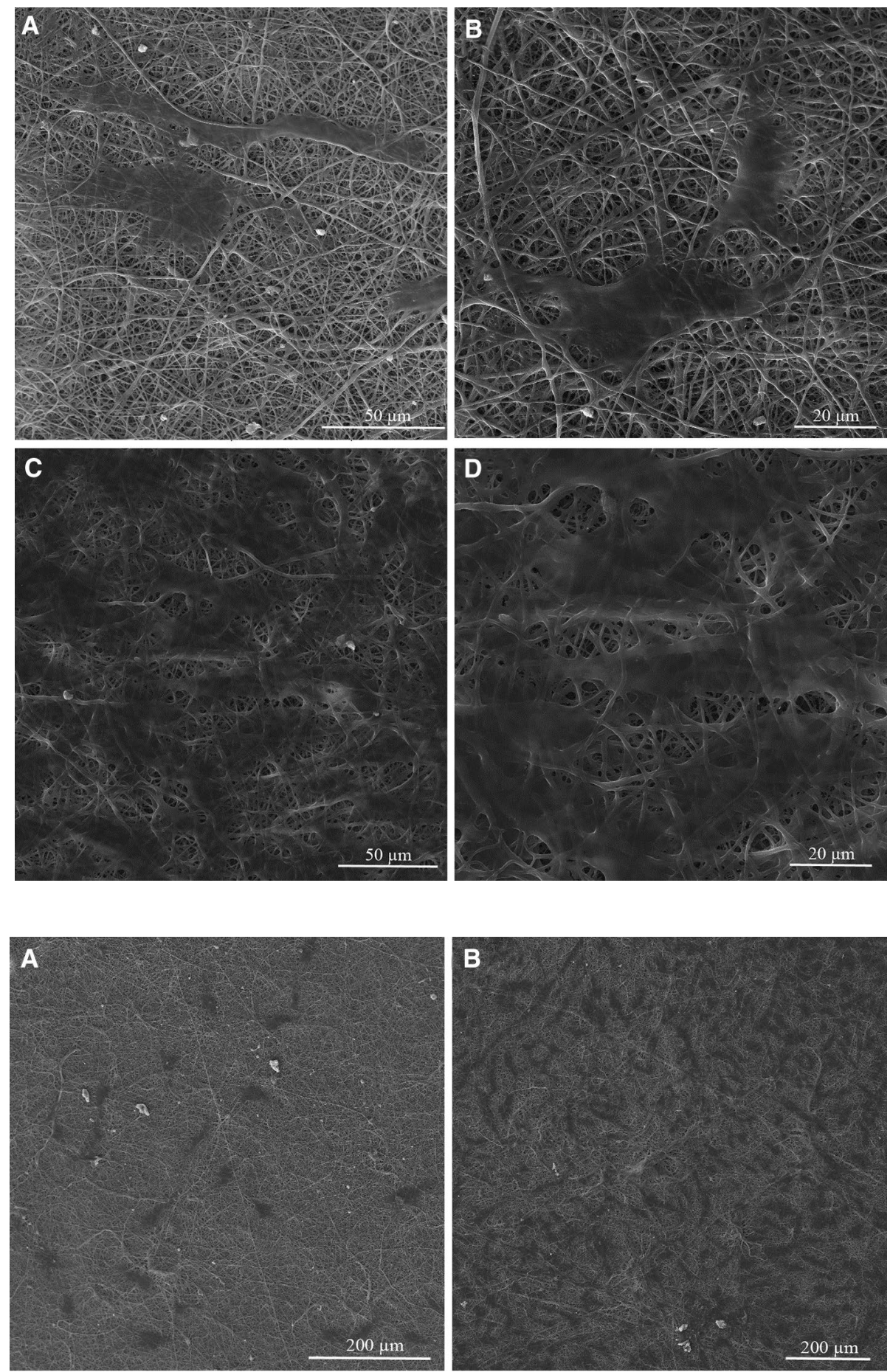

Fig. 9 Micrograph of HDF cells on electrospun Chi 30 scaffold after 1 (a) and 7 (b) days of culturing with different magnification scaffolds in culture medium were remained intact during 7 days. The electrospun gelatin/chitosan scaffolds have porosity around $92 \%$ and tensile strength of $1.1 \mathrm{MPa}$. The FTIR spectroscopy analysis demonstrated the presence of chitosan in nanofibrous structure. To assay the bioactivity of scaffolds, the attachment, morphology and proliferation of HDF cells on electrospun gelatin/chitosan scaffolds were analyzed. The morphological observation showed that HDF cells attached and spread well on gelatin/chitosan nanofibrous scaffolds exhibiting spindle-like shape. The SEM micrograph also revealed that cross-linked electrospun gelatin/chitosan blends were able to maintain their nanofibrous morphology in culture medium and provide steady physical and chemical support for cell growth. The MTS results demonstrated the significant beneficially influence of chitosan and also the nanofibrous morphological on the HDF cell 


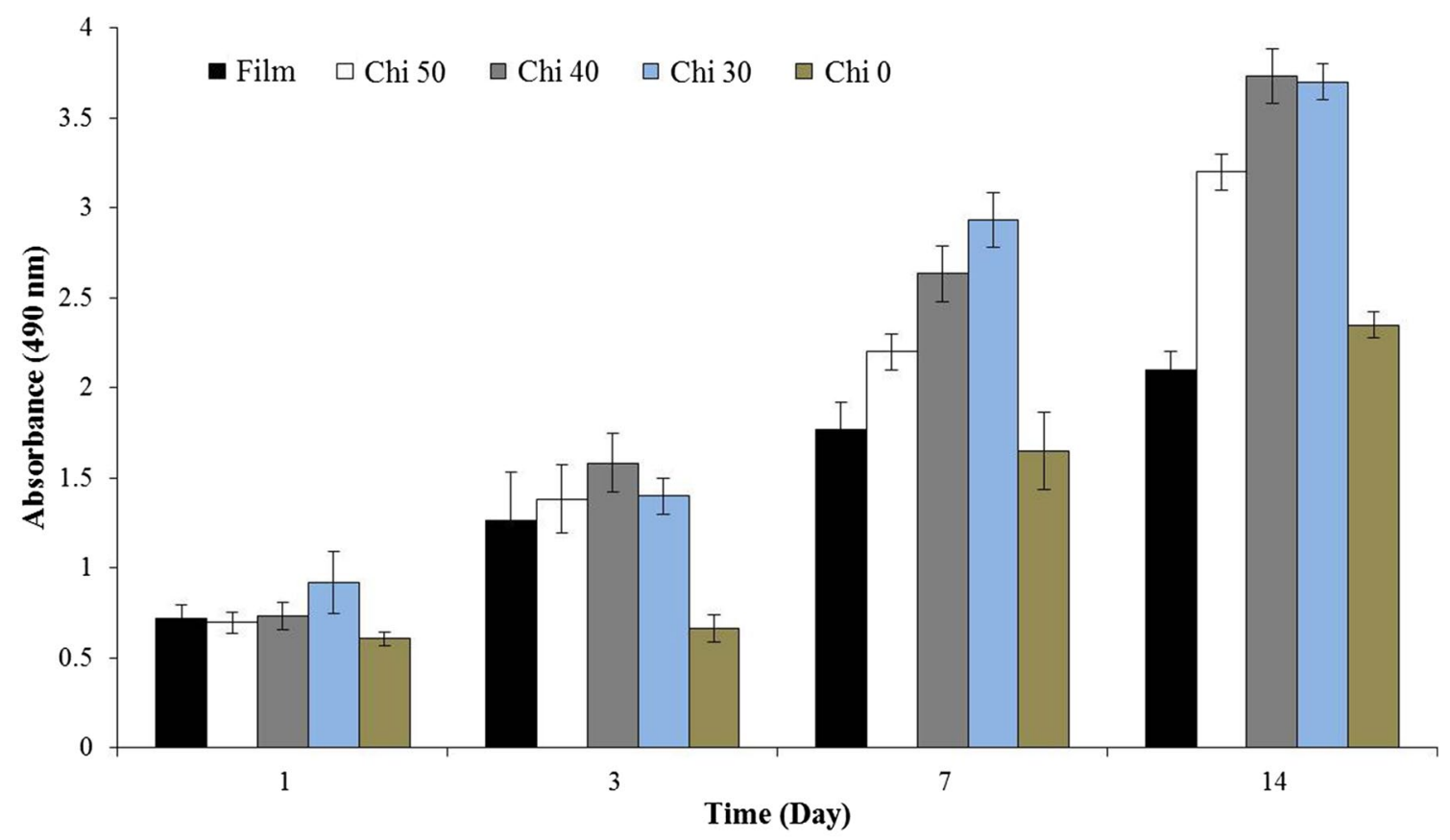

Fig. 10 MTS assay after HDF cells were cultured on nanofibrous scaffolds. Formazan absorbance expressed as a function of culture time

proliferation. Considering the MTS assay, porosity and also the mechanical property of gelatin/chitosan nanofibers with different ratios, it could be stated that Chi 30 (70/30 gelatin/ chitosan composition) has a good potential for using in skin tissue engineering application.

\section{Compliance with ethical standards}

Conflict of interest The authors declare that they have no conflict of interest.

Ethical approval This article does not contain any studies with human participants or animals performed by any of the authors.

Open Access This article is distributed under the terms of the Creative Commons Attribution 4.0 International License (http://creativeco mmons.org/licenses/by/4.0/), which permits unrestricted use, distribution, and reproduction in any medium, provided you give appropriate credit to the original author(s) and the source, provide a link to the Creative Commons license, and indicate if changes were made.

\section{References}

Al-Saidi G, Al-Alawi A, Rahman M, Guizani N (2012) Fourier transform infrared (FTIR) spectroscopic study of extracted gelatin from shaari (Lithrinus microdon) skin: effects of extraction conditions. Int Food Res J 19:1167-1173
Barikani M, Oliaei E, Seddiqi H, Honarkar H (2014) Preparation and application of chitin and its derivatives: a review. Iran Polym J 23:307-326

Barnes CP, Sell SA, Boland ED, Simpson DG, Bowlin GL (2007) Nanofiber technology: designing the next generation of tissue engineering scaffolds. Adv Drug Deliv Rev 59:1413-1433

Baxter RM et al (2013) Chitosan dressing promotes healing in third degree burns in mice: Gene expression analysis shows biphasic effects for rapid tissue regeneration and decreased fibrotic signaling. J Biomed Mater Res Part A 101:340-348

Bhardwaj N, Kundu SC (2010) Electrospinning: a fascinating fiber fabrication technique. Biotechnol Adv 28:325-347

Bin Ahmad M, Lim JJ, Shameli K, Ibrahim NA, Tay MY (2011) Synthesis of silver nanoparticles in chitosan, gelatin and chitosan/ gelatin bionano composites by a chemical reducing agent and their characterization. Molecules 16:7237-7248

Chandrasekaran AR, Venugopal J, Sundarrajan S, Ramakrishna S (2011) Fabrication of a nanofibrous scaffold with improved bioactivity for culture of human dermal fibroblasts for skin regeneration. Biomed Mater 6:015001

Chen Z, Wang P, Wei B, Mo X, Cui F (2010) Electrospun collagen-chitosan nanofiber: a biomimetic extracellular matrix for endothelial cell and smooth muscle cell. Acta Biomater 6:372-382

Chong E, Phan T, Lim I, Zhang Y, Bay B, Ramakrishna S, Lim C (2007) Evaluation of electrospun PCL/gelatin nanofibrous scaffold for wound healing and layered dermal reconstitution. Acta Biomater 3:321-330

Dabouian A, Bakhshi H, Irani S, Pezeshki-Modaress M (2018) $\beta$-Carotene: a natural osteogen to fabricate osteoinductive electrospun scaffolds. RSC Adv 8:9941-9945

Dhandayuthapani B, Krishnan UM, Sethuraman S (2010) Fabrication and characterization of chitosan-gelatin blend nanofibers for skin tissue engineering. J Biomed Mater Res Part B Appl Biomater 94:264-272

Esfandiarpour-Boroujeni S, Bagheri-Khoulenjani S, Mirzadeh H (2016) Modeling and optimization of degree of folate grafted 
on chitosan and carboxymethyl-chitosan progress. Biomaterials $5: 1-8$

Haider S, Al-Masry WA, Bukhari N, Javid M (2010) Preparation of the chitosan containing nanofibers by electrospinning chitosan-gelatin complexes. Polym Eng Sci 50:1887-1893

Heydarkhan-Hagvall S et al (2008) Three-dimensional electrospun ECM-based hybrid scaffolds for cardiovascular tissue engineering. Biomaterials 29:2907-2914

Jafari J, Emami SH, Samadikuchaksaraei A, Bahar MA, Gorjipour F (2011) Electrospun chitosan-gelatin nanofiberous scaffold: Fabrication and in vitro evaluation. Bio-Med Mater Eng 21:99-112

Jayakumar R, Prabaharan M, Sudheesh Kumar P, Nair S, Tamura H (2011) Biomaterials based on chitin and chitosan in wound dressing applications. Biotechnol Adv 29:322-337

Jiankang H, Dichen L, Yaxiong L, Bo Y, Bingheng L, Qin L (2007) Fabrication and characterization of chitosan/gelatin porous scaffolds with predefined internal microstructures. Polymer 48:4578-4588

Lai J-Y, Li Y-T, Cho C-H, Yu T-C (2012) Nanoscale modification of porous gelatin scaffolds with chondroitin sulfate for corneal stromal tissue engineering Int J. Nanomed 7:1101-1114

Mahboudi S, Pezeshki-Modaress M, Noghabi KA (2015) The study of fibroblast cell growth on the porous scaffold of gelatin-starch blend using the salt-leaching and lyophilization method. Int $\mathbf{J}$ Polym Mater Polym Biomater 64:653-659

Mao J, Zhao L, de Yao K, Shang Q, Yang G, Cao Y (2003a) Study of novel chitosan-gelatin artificial skin in vitro. J Biomed Mater Res Part A 64:301-308

Mao JS, Zhao LG, Yin YJ, Yao KD (2003b) Structure and properties of bilayer chitosan-gelatin scaffolds. Biomaterials 24:1067-1074

Martínez-Camacho AP, Cortez-Rocha MO, Castillo-Ortega MM, Burgos-Hernández A, Ezquerra-Brauer JM, Plascencia-Jatomea M (2011) Antimicrobial activity of chitosan nanofibers obtained by electrospinning. Polym Int 60:1663-1669

Modaress MP, Mirzadeh H, Zandi M (2012) Fabrication of a porous wall and higher interconnectivity scaffold comprising gelatin/chitosan via combination of salt-leaching and lyophilization methods. Iran Polym J 21:191-200

Mottaghitalab F, Hosseinkhani H, Shokrgozar MA, Mao C, Yang M, Farokhi M (2015) Silk as a potential candidate for bone tissue engineering. J Control Release 215:112-128

Nguyen T-H, Lee B-T (2010) Fabrication and characterization of crosslinked gelatin electro-spun nano-fibers. Commun Netw 2

Pant HR, Kim CS (2013) Electrospun gelatin/nylon-6 composite nanofibers for biomedical applications. Polym Int 62:1008-1013

Pezeshki-Modaress M, Rajabi-Zeleti S, Zandi M, Mirzadeh H, Sodeifi N, Nekookar A, Aghdami N (2013) Cell loaded gelatin/chitosan scaffolds fabricated by salt-leaching/lyophilization (SLL) for skin tissue engineering: in vitro and in vivo study. J Biomed Mater Res Part A
Pezeshki-Modaress M, Zandi M, Mirzadeh H (2014) Fabrication of gelatin/chitosan nanofibrous scaffold: process optimization and empirical modeling. Polym Int

Pezeshki-Modaress M, Mirzadeh H, Zandi M, Rajabi-Zeleti S, Sodeifi N, Aghdami N, Mofrad MR (2017) Gelatin/chondroitin sulfate nanofibrous scaffolds for stimulation of wound healing: in-vitro and in-vivo study. J Biomed Mater Res Part A 105:2020-2034

Pietrucha K, Marzec E (2005) Dielectric properties of the collagenglycosaminoglycans scaffolds in the temperature range of thermal decomposition. Biophys Chem 118:51-56

Qian Y-F, Zhang K-H, Chen F, Ke Q-F, Mo X-M (2011) Cross-linking of gelatin and chitosan complex nanofibers for tissue-engineering scaffolds. J Biomater Sci Polymer Edition 22:1099-1113

Rahman MM, Pervez S, Nesa B, Khan MA (2013) Preparation and characterization of porous scaffold composite films by blending chitosan and gelatin solutions for skin tissue engineering. Polym Int 62:79-86

Sadeghi A, Pezeshki-Modaress M, Zandi M (2018) Electrospun polyvinyl alcohol/gelatin/chondroitin sulfate nanofibrous scaffold: fabrication and in vitro evaluation. Int J Biol Macromol

Saeed SM, Mirzadeh H, Zandi M, Barzin J (2017) Designing and fabrication of curcumin loaded PCL/PVA multi-layer nanofibrous electrospun structures as active wound dressing. Prog Biomater 1-10

Sell SA, Wolfe PS, Garg K, McCool JM, Rodriguez IA, Bowlin GL (2010) The use of natural polymers in tissue engineering: a focus on electrospun extracellular matrix analogues. Polymers 2:522-553

Wang TW, Sun JS, Wu HC, Huang YC, Lin FH (2007) Evaluation and biological characterization of bilayer gelatin/chondroitin6-sulphate/hyaluronic acid membrane. J Biomed Mater Res Part B Appl Biomater 82:390-399

Yin Y, Ye F, Cui J, Zhang F, Li X, Yao K (2003) Preparation and characterization of macroporous chitosan-gelatin/ $\beta$-tricalcium phosphate composite scaffolds for bone tissue engineering. J Biomed Mater Res Part A 67:844-855

Zandi M, Mirzadeh H, Mayer C (2007) Effects of concentration, temperature, and $\mathrm{pH}$ on chain mobility of gelatin during early stages of gelation. Iran Polym J 16:861

Zhong S, Teo WE, Zhu X, Beuerman R, Ramakrishna S, Yung LYL (2005) Formation of collagen-glycosaminoglycan blended nanofibrous scaffolds and their biological properties. Biomacromol 6:2998-3004

Publisher's Note Springer Nature remains neutral with regard to jurisdictional claims in published maps and institutional affiliations.

\section{Affiliations}

\section{Mohamad Pezeshki-Modaress $^{1} \cdot$ Mojgan Zandi $^{2} \cdot$ Sarah Rajabi $^{3}$}

Mohamad Pezeshki-Modaress

pezeshkimodaress.m@iums.ac.ir

1 Burn Research Center, Iran University of Medical Sciences, Tehran, Iran

2 Department of Biomaterials, Iran Polymer and Petrochemical Institute, P.O. Box: 14965/159, Tehran, Iran
Department of Stem Cells and Developmental Biology, Cell Science Research Center, Royan Institute for Stem Cell Biology and Technology, ACECR, P.O. Box 19395/4644, Tehran, Iran 